\title{
An Array of Leaky Wave Antennas for Indoor Smart Wireless Access Point Applications
}

\author{
Alejandro Gil-Martínez \\ Department of Information and \\ Communications Technologies \\ Technical University of Cartagena \\ Cartagena, Spain \\ alexgil284@gmail.com
}

\author{
José Luis Gómez-Tornero \\ Department of Information and \\ Communications Technologies \\ Technical University of Cartagena \\ Cartagena, Spain \\ josel.gomez@upct.es
}

\author{
Youssef El Gholb \\ Department of Electrical Engineering \\ Faculty of Sciences and Technology \\ Sidi mohamed Ben Abdellah University \\ Fes, Morocco \\ youssef.elgholb@gmail.com \\ Najiba El Amrani El Idrissi \\ Department of Electrical Engineering \\ Faculty of Sciences and Technology \\ Sidi mohamed Ben Abdellah University \\ Fes, Morocco \\ elamrani.naj@gmail.com
}

\author{
Miguel Poveda-García \\ Department of Information and \\ Communications Technologies \\ Technical University of Cartagena \\ Cartagena, Spain \\ miguel.poveda@upct.es
}

\begin{abstract}
An array of leaky-wave antennas (LWA), designed for indoor smart wireless access points applications is presented in this work. It is designed to produce directive frequency scanning in the $\mathbf{2 . 4 5} \mathrm{Wi}-\mathrm{Fi}$ band. More particularly, it is demonstrated how three different Wi-Fi channels, namely channel \#1 (2.412 GHz), channel \#6 (2.437 GHz), and channel \#11 (2.462 GHz), can be directed towards different directions in space and offering high gain. In this way, it is proposed a simple channel-hopping technique to provide high signal levels in a wide area, and thus assure high data transmissions. The array is designed in single layer planar technology, using low-cost laminate to provide a low-profile antenna structure which can be mounted on ceilings.
\end{abstract}

Keywords- smart antenna, WiFi WLAN, reconfigurable antenna.

\section{INTRODUCTION}

Conventional access points (AP) in Wireless Local Area Networks (WLANs) such as WiFi use omnidirectional antennas to obtain good spatial coverage, but this is at the cost of wasting much energy which is radiated into directions of space where no users are present. In this sense, smart wireless AP produce beamforming to focus the signal only in the desired direction of space. Normally, these smart antennas use active circuits (RF switches, phase-shifters...) and phasedarray techniques to produce directive beams which are scanned in space [1], [2]. In particular, this active phased-array beamforming techniques are being applied in WiFi WLANs [3], using a phased-array of several monopole antennas.

In this paper we propose a totally passive smart leakyWave antenna for Wi-Fi WLANs, based on half-width microstrip (HWM) technology [4]. It provides directive beam scanning without the need of any active circuit, thus reducing the complexity, cost and power consumption if compared to active beamforming phased arrays. For that, we make use of the inherent frequency-scanning property of LWAs [5], which allow to scan a directive beam in one plane by just sweeping the frequency of the input RF signal. Moreover, we apply this to the new WiFi 802.11ax standard (also known as WiFi 6) [6], which among many new features introduces the multiuser multiple input multiple output (MU-MIMO) by using several WiFi channels simultaneously. Effectively, up to 802.11ax, one AP could only use a single WiFi channel, which was shared by all users connected to this AP. With the upcoming WiFi 6 standard, one single AP can simultaneously use different simultaneous channels, so that each WiFi user can use all the bandwidth of one channel. For instance, the Quantenna has presented a WiFi chip [7], which is being used in $\mathrm{WiFi}$ router of different companies such as american Edgewater Wireless [8], which propose a "Multi-Channel Single Radio" (MCSR) spectrum slicing using WiFi channels $\# 1$, \#6 and \#11, with respective central frequencies at 2.412 $\mathrm{GHz}, 2.437 \mathrm{GHz}$, and $2.462 \mathrm{GHz}$. In this work we demonstrate with a design how the HWM LWA technology can use this WiFi MU-MiMo channel slicing to provide six simultaneous beams covering a wide angular field of view of $90^{\circ} \mathrm{deg}$, with a gain of $15 \mathrm{dBi}$.

\section{ANTENNA DESIGN}

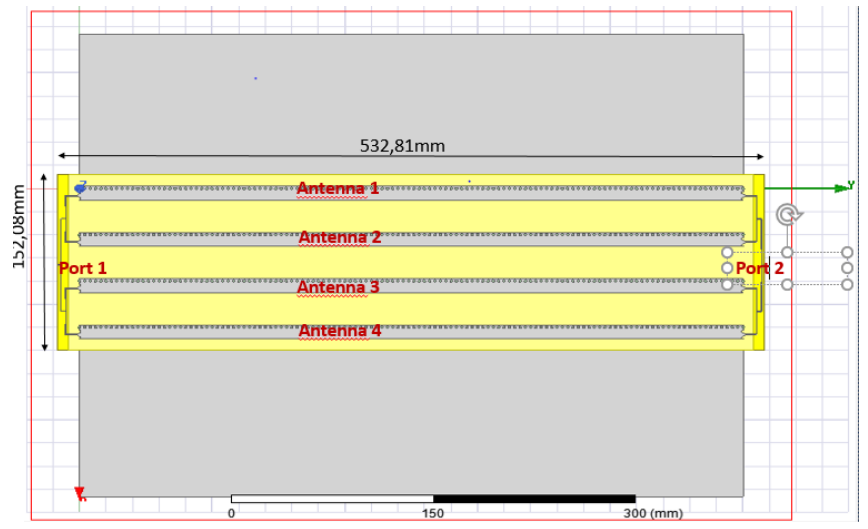

Fig. 1. Scheme of the proposed smart WiFi 6 LWA array.

The layout of the proposed smart WiFi 6 antenna is sketched in Fig. 1. It consists of four half-width microstrip (HWM) leaky-wave antennas (LWAs) [4], which are arranged in parallel and fed by a 1:4 equal power divider. The antenna has two input ports, which can be connected to a $2 \times 2$ MiMo WiFi router which can switch between any of these two ports. As it will be shown, each port is able to generate three simultaneous directive beams when using the aforementioned WiFi channels \#1, \#6 and \#11. Thus, the antenna will create a total of six directive beams when connected to a WiFi 6 router 
with this channel slicing as [9]. Low-cost AD1000 substrate is used in the design, with thickness $\mathrm{H}=0.508 \mathrm{~mm}, \mathrm{Er}=10.068$ and $\tan \delta=0.0018$. The total dimension of the planar antenna is $53,28 \mathrm{~cm} \times 15,21 \mathrm{~cm}$. In order to increase even more the directivity of the smart array, it is placed on a bigger metallic ground plane, which prevents radiation to the backward direction (-z direction in Fig. 1).

All the HWM LWAis are identical and have been designed to provide the desired frequency-scanning behavior, obtaining the dimensions shown in Fig. 2. Particularly, the radiating length of the HWM antennas is $50 \mathrm{~cm}$, so to obtain directive scanned beam with half-power beamwidth of $20^{\circ}$ in the 2.45 $\mathrm{GHz}$ band. For that, the width of the strip patches is set to $1.008 \mathrm{~cm}$ as shown in Fig. 2. Metallic vias with a radius of 1 $\mathrm{mm}$ and separated a distance of $4 \mathrm{~mm}$ used in the nonradiating side of the HWM. Finally, width-tapered microstrip line with an inset is used to optimize the input matching at each individual antenna, with dimensions summarized in Fig. 2. Adjacent radiating faces of each HWM LWA are separated at a lateral distance of $3 \mathrm{~cm}$. A 1:4 equal-power microstrip divider formed by two cascaded 1:2 sections is used to feed the four elements of the array. The transmission line dimensions are summarized in Fig. 3. The array is completely symmetrical and thus similar feeding networks are used for both input ports, as shown in Fig. 1.

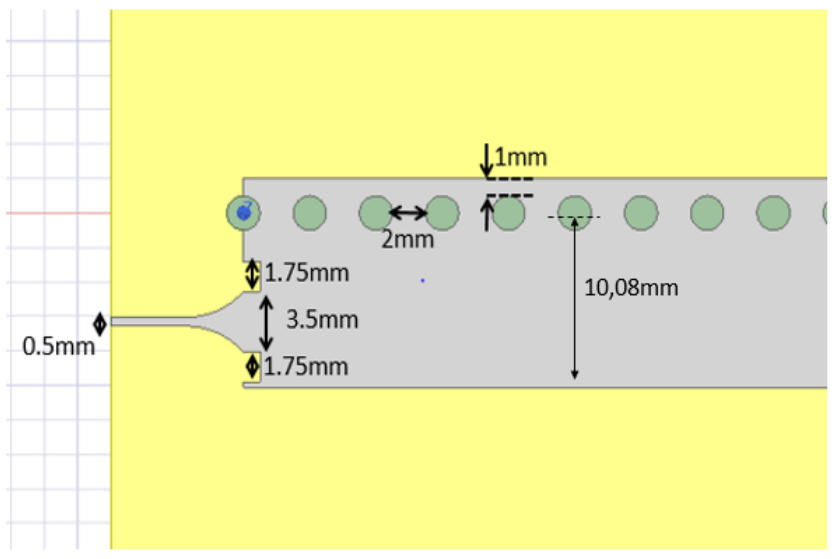

Fig. 2. Scheme and dimensions of individual LWA element.

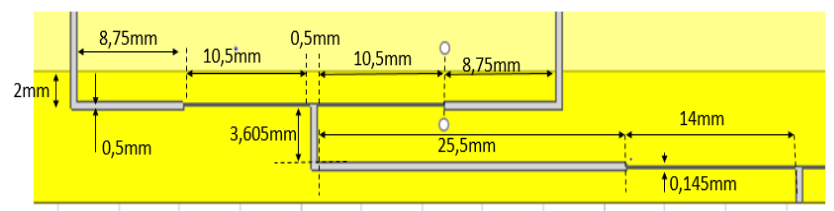

Fig. 3. Scheme and dimensions of 1:4 feeding network

The obtained input matching (S11) and coupling between ports (S21) are obtained using the commercial full-wave electromagnetic simulator HFSS and plotted in Fig. 4. The three Wi-Fi channels of interest (\#1, \#6 and \#11) are shown in Table II, and their respective matching and coupling values are summarized in Table I. As it can be seen in the WiFi frequency band the S11 parameter for each chosen channel is down to $8 \mathrm{~dB}$ indicating that almost the $1 \%$ of the input power is reflected in connectors and since the antennas are symmetrical this happens in both ports equally. On the other hand, the S21 parameter is down to $-10 \mathrm{~dB}$ in all the interest frequencies, this means that the rest of the input power which enter inside the antenna is radiated so the power that reaches port two is largely dissipated avoiding in this way reflected waves inside the antenna and wherefore the isolation between ports is good.

The beam in ZX plane (E-Plane) is narrow due to the array configuration of the four HWM LWA, thus, the gain in this plane is significantly high, as it can be seen in Fig.5. This way an antenna with high directive in both planes can be achieved. E-Plane and H-Plane, so that we have an antenna that allows high spatial resolution.

The $3 \mathrm{D}$ radiation patterns are shown in next three Figures whereby the directive frequency scanning in WiFi $2.45 \mathrm{GHz}$ band and the high Gain and Directivity aim are obtained as it can be seen. These results are obtained by feeding the antenna by port one, so the radiating angles are the positive ones. Due to this behaviour of the antenna, reconfigurable capability is achieved.

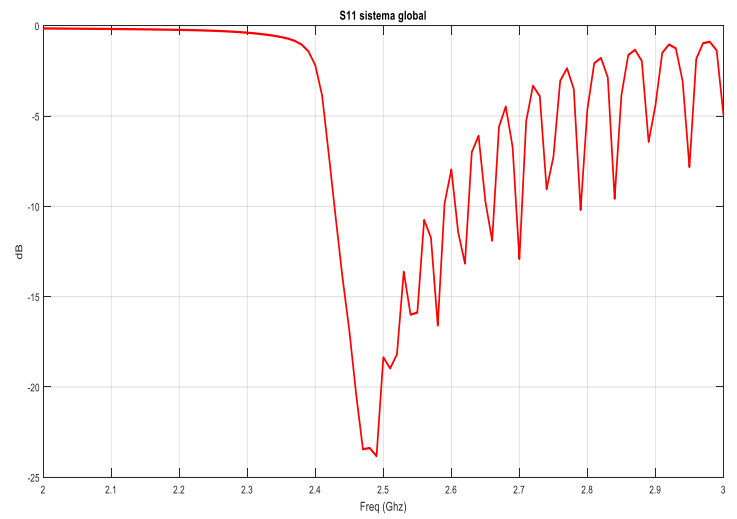

Fig. 4. Simulated S11 and S21 parameters.

TABLE I. PORTS MATCHING AND ISOLATION AT WI-Fi CHANNELS

\begin{tabular}{|l|c|c|c|}
\hline Channel & $\begin{array}{c}\# 1 \\
(2.412 \mathrm{GHz})\end{array}$ & $\begin{array}{c}\# 6 \\
(2.437 \mathrm{GHz})\end{array}$ & $\begin{array}{c}\# 11 \\
(2.462 \mathrm{GHz})\end{array}$ \\
\hline Matching & $-8.27 \mathrm{~dB}$ & $-20.5 \mathrm{~dB}$ & $-13.04 \mathrm{~dB}$ \\
\hline Coupling & $-33.98 \mathrm{~dB}$ & $-14.16 \mathrm{~dB}$ & $-9.98 \mathrm{~dB}$ \\
\hline
\end{tabular}

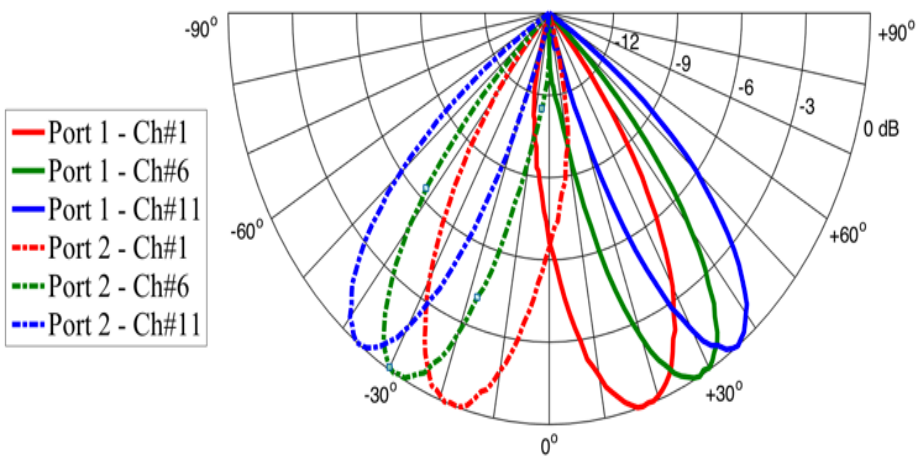

Fig. 5. Simulated radiation patterns in the scanning ZY plane. 


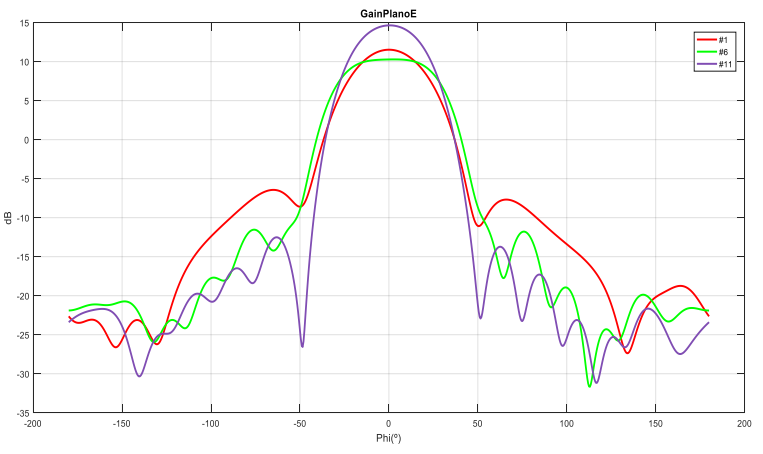

Fig. 6. Simulated radiation patterns in the perpendicular ZX plane.

TABLE II. SCANNED BEAM PRoperties

\begin{tabular}{|l|c|c|c|}
\hline Channel & $\begin{array}{c}\# 1 \\
(2.412 \mathrm{GHz})\end{array}$ & $\begin{array}{c}\# 6 \\
(2.437 \mathrm{GHz})\end{array}$ & $\begin{array}{c}\# 11 \\
(2.462 \mathrm{GHz})\end{array}$ \\
\hline $\begin{array}{l}\text { Scanning } \\
\text { angle in ZY }\end{array}$ & $11.5^{\circ}$ & $26^{\circ}$ & $37.5^{\circ}$ \\
\hline ZY HPBW & $18.75^{\circ}$ & $12.75^{\circ}$ & $13.5^{\circ}$ \\
\hline ZX HPBW & $40^{\circ}$ & $58^{\circ}$ & $40^{\circ}$ \\
\hline Peak gain & $13.56 \mathrm{dBi}$ & $15.35 \mathrm{dBi}$ & $15.21 \mathrm{dBi}$ \\
\hline $\begin{array}{l}\text { Radiation } \\
\text { efficiency }\end{array}$ & $69.6 \%$ & $70.0 \%$ & $67.1 \%$ \\
\hline
\end{tabular}
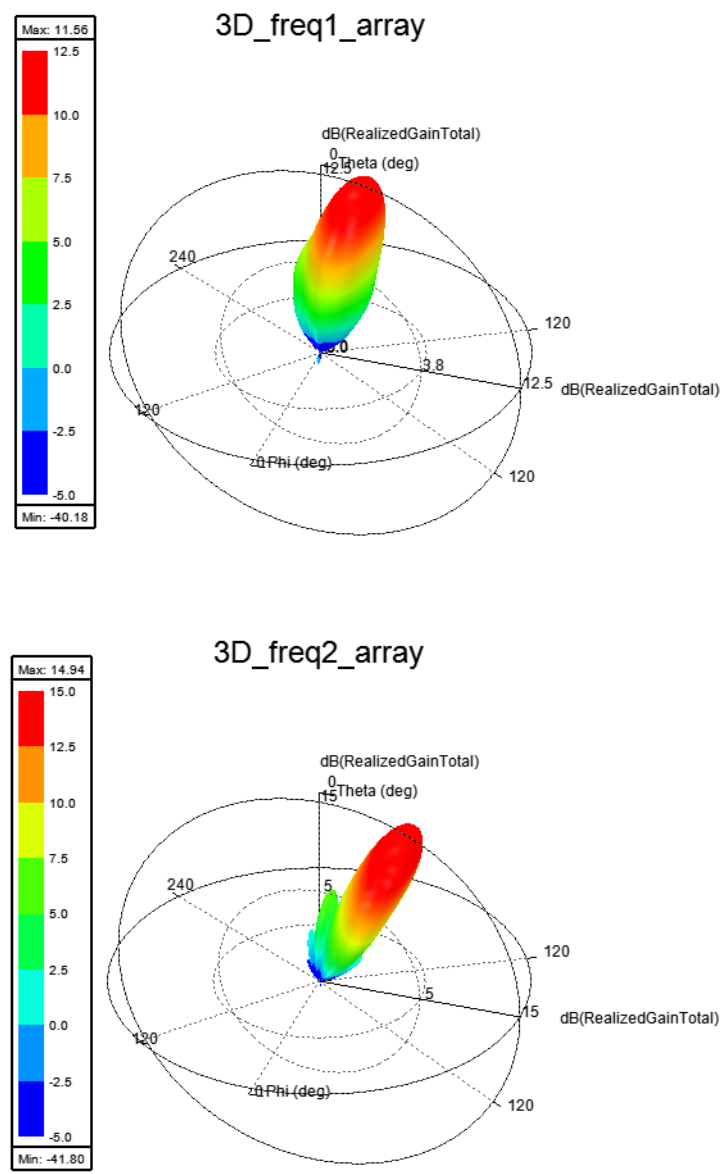

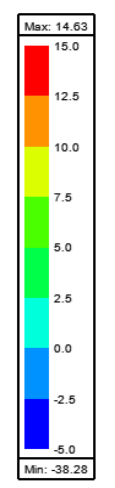

3D_freq3_array

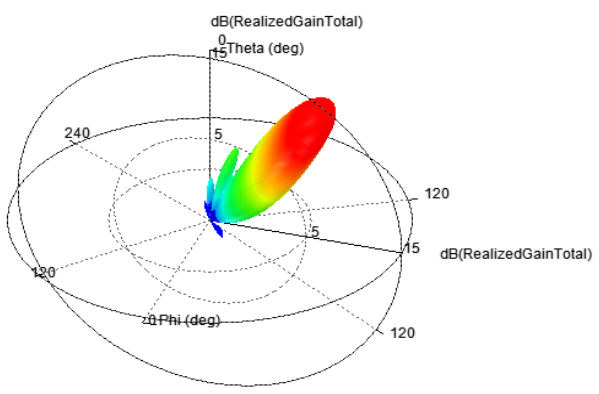

III. CONCLUSIONS

We have presented the design of an array of leaky-wave antennas (LWAs) to generate six simultaneous scanned directive beams when connected to a MU MiMO 802.11ax WiFi router. It has been designed to match the specifications of simultaneous channel-slicing using WiFi channels \#1, \#6 and \#11, so to conceive a novel type of smart access point, which provide unprecedent $15 \mathrm{dBi}$ gain over a wide field of view of $90^{\circ}$, and dispensing for any type of active, powerconsuming beamforming technique. We believe this type of frequency-beamforming using passive LWAs is very attractive due to the totally passive, low-profile planar structure and simple directive-scanning mechanism, which reduce the overall smart access point cost while providing high electromagnetic performance. In future works, the overall smart WiFi AP performance will be tested.

\section{ACKNOWLEDGMENT}

This work has been supported by Spanish national projects TEC2016-75934-C4-4-R and European Erasmus Grant KA107 Morocco.

\section{REFERENCES}

[1] Z. Hao, H. Wang and W. Hong, "A Novel Planar Reconfigurable Monopulse Antenna for Indoor Smart Wireless Access Points' Application," IEEE Transactions on Antennas and Propagation, vol. 64, no. 4, pp. 1250-1261, April 2016.

[2] J. Keh, M. Chou, Z. Zhang, Q. An and W. Liao, "A BeamformingNetwork-Based Four-Squint-Beam Array Antenna for Ceiling-Mount Access Point," IEEE Antennas and Wireless Propagation Letters, vol. 18, no. 4, pp. 707-711, April 2019.

[3] "Beamforming in 802.11ac", chapter 4 de 802.11ac: A Survival Guide. por Matthew S. Gast, Ed. O'Reilly Media, Inc, August 2013, ISBN: 9781449357702, available online at:

https://www.oreilly.com/library/view/80211ac-asurvival/9781449357702/ch04.html

[4] G. Zelinski, G. Thiele, M. Hastriter, M. Havrilla, and A. Terzuoli, "Half width leaky wave antennas," IET Microw. Antennas Propag., vol. 1, no. 2, pp. 341-348, Apr. 2007.

[5] A. A. Oliner, "Leakage from higher modes on microstrip line with application to antennas," Radio Sci., vol. 22, pp. 907-912, Nov. 1987.

[6] Wi-Fi CERTIFIED $6^{\mathrm{TM}}$ coming in 2019, available online at: https://www.wi-fi.org/news-events/newsroom/wi-fi-certified-6coming-in-2019

[7] QSR10GU-AX Plus Chipset Solution, available online at: http://www.quantenna.com/products/qsr10g-ax/

[8] Edgewater Wireless Wi-Fi SPECTRUM SLICING, available online at: https://www.edgewaterwireless.com/mcsr-explained/

[9] EAP3030 3-channel WiFi3 ${ }^{\mathrm{TM}}$ AP - INDOOR, available online at: https://www.edgewaterwireless.com/wpcontent/uploads/2016/04/EAP3030-Indoor-Final.pdf.zip 